\title{
Relações entre Preconceito e Crenças sobre Diversidade Sexual e de Gênero em Psicólogos/as Brasileiros/as
}

\author{
Icaro Bonamigo Gaspodini ${ }^{1}$

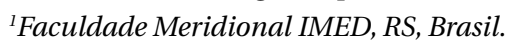

\author{
Denise Falcke ${ }^{2}$ \\ ${ }^{2}$ Universidade do Vale do Rio dos Sinos, RS, Brasil.
}

Resumo: Examinar as crenças de psicólogos/as sobre indivíduos e grupos atingidos pelo preconceito contribui para a preparação do/a profissional no atendimento a esse público. Neste estudo, investigou-se a relação entre preconceito e crenças sobre diversidade sexual e de gênero em psicólogos/as brasileiros/as. Um questionário online foi respondido por 497 profissionais, entre 22 e 69 anos $(M=34,52 ; D P=9,57)$, ligados/as a 22 dos 23 Conselhos Regionais de Psicologia. Utilizou-se a Escala de Preconceito contra Diversidade Sexual e de Gênero Revisada para mensurar o preconceito e a Escala de Crenças sobre a Natureza da Homossexualidade para investigar crenças sobre homossexualidade, a qual foi replicada para também investigar crenças sobre bissexualidade e transexualidade. Observaram-se índices baixos de preconceito e a predominância de crenças psicossociais para compreender a diversidade sexual e de gênero. O tipo de crença que melhor explicou a ocorrência de preconceito foi de base psicológica, representado pelas atribuições causais de perversão, má resolução de conflitos com figuras parentais e abusos sexuais sofridos na infância. Além disso, embora com tamanhos de efeito pequenos, foram encontradas correlações positivas de preconceito com a perspectiva teórica da psicanálise e orientação psicanalítica, com a modalidade de avaliação psicológica e/ou psicodiagnóstico e com a área de atuação em neurociência do comportamento. Tendo em vista a população estudada, ainda que os índices tenham sido baixos, considera-se preocupante a manifestação de preconceito e, em especial, o fato de crenças psicológicas terem sido as que melhor explicaram os índices mais elevados de preconceito.

Palavras-chave: Preconceito, Homofobia; Atuação do Psicólogo; Psicólogos. 


\title{
Relations Between Prejudice and Beliefs About Sexual and Gender Diversity Among Brazilian Psychologists
}

\begin{abstract}
Examining psychologists' beliefs about individuals and groups who are victims of prejudice is a way to enhance these professionals' training when working with this public. The objective of this study was to investigate relations between prejudice and beliefs about the nature sexual and gender diversity among Brazilian psychologists. A total of 497 professionals responded to an online questionnaire. Participants' ages ranged from 22 to 69 years old $(M=34.52 ; S D=9.57$ ) and they were associated to 22 out of 23 Regional Psychology Councils. We used the Revised Scale of Prejudice Against Sexual and Gender Diversity to measure prejudice and the Scale of Beliefs About the Nature of Homosexuality to investigate beliefs about homosexuality, which was replicated to investigate beliefs about bisexuality and transsexuality as well. Low rates in extreme prejudice were observed and psychosocial beliefs about sexual and gender diversity were predominant. Psychological beliefs best explained the occurrence of prejudice, represented by the following causal attributions: perversion of normal behavior, unsatisfactory conflict resolution with parental figures, and sexual abuse in childhood. Besides, although showing low size effects, positive correlations were found between the theoretical perspective of psychoanalysis, the modality of psychological assessment and/or psychodiagnosis, and the area of behavioral neuroscience. In view of the population studied, we consider apprehensive that, even with low rates, extreme prejudice scores were above the minimum and, especially, the fact that psychological beliefs best explained the highest scores on extreme prejudice against sexual and gender diversity.
\end{abstract}

Keywords: Prejudice, Homophobia, Psychologist Performance, Psychologists.

\section{Relaciones entre Preconcepto y Creencias sobre Diversidad Sexual y de Género en Psicólogos/as Brasileños/as}

Resumen: Examinar las creencias de psicólogos/as sobre individuos y grupos afectados por el preconcepto contribuye a la preparación del profesional en la atención a ese público. En este estudio, se investigó la relación entre preconceptos y creencias sobre diversidad sexual y de género en psicólogos/as brasileños/as. Un cuestionario on line fue respondido por 497 profesionales, con edades entre 22 y 69 años $(M=34,52, D P=9,57)$, relacionados/das a 22 de los 23 Consejos Regionales de Psicología. Se utilizó la Escala de Preconcepto contra Diversidad Sexual y de Género Revisada para medir el preconcepto y la Escala de Creencias sobre la Naturaleza de la Homosexualidad para investigar creencias sobre homosexualidad, la cual fue replicada para también investigar creencias sobre bisexualidad y transexualidad. Se observaron bajos índices de preconcepto y la predominancia de creencias psicosociales para comprender la diversidad sexual y de género. El tipo de creencia que mejor explicó la ocurrencia de prejuicio fue de base psicológica, representado por las atribuciones causales de perversión, mala resolución de conflictos con figuras parentalesy abusos sexuales sufridos en la infancia. Además, aunque con tamaños de efecto pequeño, se encontraron correlaciones positivas de preconcepto con la perspectiva teórica del psicoanálisis y orientación psicoanalítica, con la modalidad de evaluación psicológica y/o psicodiagnóstico y con el área de actuación en neurociencia del comportamiento. En vista de la población estudiada, aunque los índices han sido bajos, se considera preocupante la manifestación de preconcepto y, en especial, el hecho de que creencias psicológicas hayan sido las que mejor explicaron los índices más elevados de preconcepto.

Palabras clave: Preconcepto, Homofobia, Actuación del Psicólogo, Psicólogos. 
Em diversos países, organizações de Psicologia realizam ações que visam reduzir preconceito, discriminação e violência contra pessoas lésbicas, gays, bissexuais, travestis e transexuais - LGBT (American Psychological Association, 2009; Australian Psychological Society, 2007; Canadian Psychological Association, n.d., Conselho Federal de Psicologia, 2011, n.d.). Parte dessas ações consiste na tomada de consciência de que a Psicologia teve participação no processo histórico de considerar como psicopatologia as diversas manifestações da diversidade sexual e de gênero (Costa, Santos, Rodrigues, \& Nardi, 2009; Jesus, 2013). Para Costa e Nardi (2015), o preconceito contra diversidade sexual e de gênero pode ser visto como um conjunto de atitudes e crenças por meio das quais os indivíduos reproduzem modelos considerados ideais de sexualidade.

Tratamentos "conversivos" ou "reparativos" para homossexuais foram oferecidos por profissionais da psiquiatria, Psicologia e psicanálise (Jesus, 2013), o que incluía o uso de eletrochoque e terapias hormonais (Daniel, \& Baudry, 1977). Sobre os efeitos dessas chamadas "terapias", a American Psychological Association (2009) publicou relatório incluindo depressão, ansiedade e tendência suicida. A Organização Mundial da Saúde considerou esses tratamentos como ameaças graves à saúde mental, ao bem-estar e à vida (Pan American Health Organization, n.d.; World Health Organization, 2012). No entanto, segundo Shipherd (2015), essas práticas ainda estão ocorrendo em diversos países. No Brasil, por exemplo, em setembro de 2017, uma decisão liminar proferida no Distrito Federal acatou em partes uma ação popular contrária à Resolução no 01/99 do Conselho Federal de Psicologia, abrindo possibilidade para práticas prejudiciais tais como as chamadas "terapias de reversão sexual". Movida por um grupo específico psicólogos/as, essa prática viola os direitos humanos e contraria décadas de pesquisas científicas sobre o tema (Conselho Federal de Psicologia, 2017). Ainda que venha a ser revertida, a decisão tem implicações prejudiciais à profissão como um todo, além de ameaçar a saúde de pessoas que são levadas a esse tipo de serviço e que não tenham condições para compreender as implicações ideológicas subjacentes aos mesmos.

Ainda que casos mais extremos - como psicólogos/as que oferecem tratamentos de "cura" da homossexualidade - não sejam comuns, ressalta-se que o preconceito passou a assumir formas mais veladas, sutis ou camufladas na contemporaneidade (For- miga, 2007; Formiga, \& Silva Neta, 2009; Pettigrew, \& Meertens, 1995, 2001). Isso pode ser explicado porque normas sociais antipreconceito, como leis e diretrizes institucionais, passaram a influenciar as pessoas a inibirem suas expressões discriminatórias contra alguns grupos-alvo (Pereira, Monteiro, \& Camino, 2009a). No entanto, os grupos minoritários que ainda não alcançaram semelhante proteção social, são atingidos de maneira mais explícita (Pereira et al., 2009a).

Nesse sentido, Pereira, Monteiro e Camino (2009b) investigaram se normas antipreconceito reduzem a sua expressão contra homossexuais. Descobriu-se que normas antipreconceito podem reduzir o preconceito flagrante, mas não o sutil, pois a expressão de emoções negativas entre participantes diminuiu, enquanto que a expressão de emoções positivas permaneceu estável (Pereira et al., 2009b). Ainda assim, a redução de preconceito flagrante como consequência de normas sociais colabora para a redução das estatísticas de violência contra a população LGBT. No Brasil, as primeiras estimativas surgiram em 2011, em relatório sobre denúncias de violações de direitos humanos contra pessoas LGBT (Brasil, 2011). No segundo relatório, um ano depois, as quase 10 mil denúncias representavam praticamente o dobro do ano anterior, sendo as travestis as mais afetadas (Brasil, 2012).

A forma como as pessoas organizam modelos explicativos sobre a diversidade sexual e de gênero tem implicações relevantes no preconceito que manifestam, porque as representações sociais sobre a natureza de grupos minoritários servem como justificativa para o preconceito (Pereira, Torres, Pereira, \& Falcão, 2011). Esses modelos influenciam a maneira como as pessoas reagem frente a indivíduos e grupos aos quais foram atribuídas determinadas características sociais como se fossem naturais, um mecanismo conhecido como essencialismo psicológico (Medin, \& Ortony, 1989; Rothbart, \& Taylor, 1992).

Com base nessa perspectiva, Lacerda, Pereira e Camino (2002) ofereceram um modelo de crenças que as pessoas mantêm sobre a natureza da homossexualidade. Pelo senso comum, atribuem-se causas à homossexualidade, que podem sem divididas em cinco tipos: (1) crenças religiosas - desobediência a Deus, falta de fé ou fraqueza espiritual para resistir a tentações; (2) crenças ético-morais - desrespeito às normas que regulam o comportamento sexual, falta de caráter ou alteração de valores morais; (3) crenças biológicas - disfunções hormonais, problemas here- 
ditários ou má formação no período da gestação; (4) crenças psicológicas - perversão do comportamento sexual normal, resolução insatisfatória de conflitos com figuras parentais e abusos sexuais sofridos na infância; (5) crenças psicossociais - modo como se forma a identidade da pessoa, práticas culturais de cada sociedade ou preferência da pessoa por essa orientação sexual (Pereira et al., 2009b).

Uma pesquisa mostrou que crenças em uma natureza biológica e psicossocial, bem como a descrença em uma natureza ético-moral da homossexualidade, estão associadas a preconceito sutil (Pereira et al., 2011). O preconceito flagrante associou-se com o contrário: descrença na natureza biológica e psicossocial e crença na natureza ético-moral da homossexualidade (Pereira et al., 2011). Outro estudo também mostrou que esportistas com crenças de natureza ético-moral sobre a homossexualidade manifestam mais atitudes preconceituosas do que os baseados em crenças psicossociais (Pereira, Alfaia, Souza, \& Lima, 2014).

Os itens indicadores de crenças de base psicológica, presentes no instrumento de A. Pereira et al. (2009b), são avaliados pela concordância do respondente em relação às causas da homossexualidade estarem relacionadas com abusos sexuais sofridos na infância, com perversão do comportamento sexual normal e com má resolução de conflitos com as figuras parentais. Se atribuições causais como essas ainda são ensinadas nos cursos de psicologia (Borges, Canuto, Oliveira, \& Vaz, 2013), torna-se relevante a investigação do preconceito nessa área. No entanto, no Brasil, os estudos são raros. Após buscas em bases de dados científicas, não foram encontrados estudos sobre essa temática envolvendo psicólogos/as no contexto brasileiro.

Algumas investigações envolveram estudantes universitários/as de Psicologia. A partir de uma tipologia de três grupos (preconceituosos flagrantes, preconceituosos sutis e não preconceituosos), Lacerda et al. (2002) mostraram que preconceituosos flagrantes se opuseram a explicações psicossociais e aderiram às explicações ético-morais e religiosas (estudantes de engenharia). Estudantes de Psicologia receberam a classificação "não preconceituosos" e aderiram a explicações psicossociais, enquanto que preconceituosos sutis aderiram a explicações biológicas e psicológicas (estudantes de medicina) (Lacerda et al., 2002). Outro estudo mostrou resultados diferentes: estudantes em fase final dos cursos de Psicologia e de direito foram comparados acerca de suas atitudes frente à adoção de crianças por casais homossexuais (Araújo, Oliveira, Souza, \& Castanha, 2007). Estudantes de Psicologia mencionaram que este tipo de adoção pode acarretar em "distúrbios psicológicos" na criança, enquanto que estudantes de direito apontaram para os possíveis "problemas morais" (Araújo et al., 2007).

A pesquisa de Camino e Pereira (2000) envolveu professores/as de Psicologia de universidades brasileiras e relacionou suas crenças sobre a natureza da homossexualidade com suas atitudes frente à Resolução $n^{\circ} 01 / 99$, que regulamenta a atuação em relação à orientação sexual. Atitudes mais negativas em relação à resolução estiveram associadas a representações da homossexualidade como um sintoma psicológico ou uma disfunção biológica (Camino, \& Pereira, 2000). Nesse sentido, ao investigar as pesquisas brasileiras em Psicologia no campo das homossexualidades, desde a criação da Resolução n ${ }^{\circ}$ 01/99 até o ano de 2010, Sposito (2015) reuniu informações como principais centros de pesquisa, perspectivas teórico-metodológicas, focos de estudo e concepções acerca da atuação do/a profissional. Observou que elas estavam concentradas no eixo sudeste/sul do Brasil, tendo identificado uma perspectiva despatologizante na maioria delas. No entanto, os estudos que comunicavam alguma intervenção psicológica no âmbito das homossexualidades mostraram-se, na maioria, relatos de atitudes patologizantes, que denotavam algum tipo de preconceito.

Com base nesses pressupostos, o presente estudo se justifica tendo em vista a presença de ideias associadas ao preconceito na Psicologia, bem como a escassez de pesquisas sobre o tema no Brasil, especialmente na população de psicólogos/as. Sendo assim, o objetivo foi investigar associações entre o preconceito e as crenças sobre a natureza da homossexualidade, bissexualidade e transexualidade nesses/as profissionais.

\section{Método}

\section{Delineamento}

Trata-se de um estudo quantitativo, transversal e explicativo.

\section{Participantes}

Psicólogos/as residentes no Brasil compuseram esta amostra não probabilística. Cerca de 2,5 mil convites foram enviados por e-mail e compartilhados em redes sociais para universidades, instituições de for- 
mação e empresas de Psicologia. O Conselho Regional de Psicologia do Rio Grande do Sul divulgou o convite em sua página oficial e o do Espírito Santo convidou todos os cadastrados por mala direta. Ao todo, participaram 497 psicólogos/as, com idades entre 22 e 69 anos $(M=34,52 ; D P=9,57)$, ligados a 22 dos 23 Conselhos Regionais de Psicologia. Na Tabela 1, apresentam-se as principais características da amostra.

\section{Instrumentos e materiais}

\section{Dados sociodemográficos e características profissionais}

Para coletar dados sociodemográficos, criou-se um questionário para que o/a respondente informasse idade, gênero, orientação sexual, região de moradia, formação completa e em andamento. Para as características profissionais, além da região e do tempo de atuação, o/a

\section{Tabela 1}

Características da amostra.

\begin{tabular}{lcc}
\hline Variável & $\%$ & $n$ \\
\hline Gếlida & & \\
$\quad$ Feminino & 79,7 & 396 \\
$\quad$ Masculino & 19,5 & 97 \\
$\quad$ Outros & 0,8 & 4 \\
Local de moradia & & \\
$\quad$ Capital & 41,9 & 208 \\
$\quad$ Interior & 41,1 & 204 \\
$\quad$ Região metropolitana & 16,9 & 84 \\
Região de atuação & & \\
$\quad$ Sul & 67,4 & 317 \\
Sudeste & 21,7 & 102 \\
$\quad$ Nordeste & 6,8 & 32 \\
Centro-Oeste & 2,8 & 13 \\
$\quad$ Norte & 1,3 & 6 \\
Tempo de atuação & & \\
Entre alguns meses até 10 anos & 70,6 & 346 \\
$\quad$ Mais de dez anos & 29,4 & 144 \\
Formação completa & & \\
$\quad$ Especialização & 40,2 & 198 \\
$\quad$ Mestrado Acadêmico & 25,0 & 123 \\
$\quad$ Graduação & 23,4 & 115 \\
Formação em andamento & & \\
$\quad$ Nenhuma & 43,8 & 208 \\
Especialização & 23,6 & 112 \\
Mestrado Acadêmico & 14,1 & 67 \\
Doutorado e Pós-Doutorado & 13,1 & 62 \\
\hline$\quad$ & \\
\hline
\end{tabular}

participante deveria indicar, em uma escala de 5 pontos $(0 \%, 25 \%, 50 \%, 75 \%$ e $100 \%)$, o quanto seu trabalho era representado naquele momento por cada uma das 10 perspectivas teóricas, oito modalidades de trabalho e 21 áreas de atuação listadas no questionário. As perspectivas teóricas foram esquizoanálise, gestalt, humanismo e existencialismo, psicanálise e orientação psicanalítica, psicodrama, Psicologia cognitiva, Psicologia comportamental, Psicologia cognitivo-comportamental, Psicologia positiva e Psicologia sistêmica. As modalidades de trabalho foram avaliação psicológica e/ou psicodiagnóstico, psicoterapia individual, psicoterapia de casal, psicoterapia de família, psicoterapia em grupo, problemas de aprendizagem, ludoterapia e psicoterapia psicomotora. As áreas de atuação foram listadas na Tabela 2, na sessão de apresentação dos resultados.

\section{Crenças sobre homossexualidade, bissexualidade e transexualidade}

Crenças sobre a homossexualidade foram medidas pela Escala de Crenças sobre a Natureza da Homossexualidade (Pereira et al., 2009b). A versão brasileira foi criada por Lacerda et al. (2002), no entanto, após contato com autor responsável, optou-se por utilizar a versão portuguesa da escala, por ser a mais recente, validada por Pereira et al. (2009b). Ela é composta por 15 itens distribuídos em escalas estilo Likert de 7 pontos $(1=$ discordo totalmente a 7 = concordo totalmente). Todos os itens iniciam pela oração "A(s) causa(s) da homossexualidade [...]" e são seguidos de alguma uma explicação. Sua estrutura fatorial indica cinco tipos de crenças sobre a homossexualidade: crenças religiosas, ético-morais, biológicas, psicológicas e psicossociais. Não foi encontrado o alfa de Cronbach da escala no estudo de validação. No presente estudo, o valor encontrado foi $\alpha=0,65$. Para medir os tipos de crenças sobre bissexualidade, essa palavra foi empregada em substituição à "homossexualidade" nos itens da escala de Pereira et al. (2009b). Encontrou-se um alfa de Cronbach de 0,68 neste estudo. O mesmo foi feito com a palavra "transexualidade", no entanto, foram alteradas as expressões "comportamento sexual" por "comportamento de gênero" (item 3) e “orientação sexual” por "expressão de gênero" (item 8). Neste estudo, o alfa de Cronbach foi de 0,66.

\section{Preconceito contra diversidade sexual e de gênero \\ A Escala de Preconceito contra Diversidade Sexual e de Gênero Revisada - EPDSG-R, desenvolvida por Costa, Peroni, Camargo, Pasley e Nardi (2015a) e revi-}


sada por Costa, Machado, Bandeira e Nardi (2016), foi utilizada com a permissão do autor responsável. A escala fornece uma medida culturalmente sensível de manifestação extrema de preconceito contra diversidade sexual e de gênero (Costa et al., 2016; Costa et al., 2015a) e foi desenvolvida a partir de uma revisão sistemática sobre homofobia e sexismo (Costa, Peroni, Bandeira, \& Nardi, 2013a) e outra sobre instrumentos que mediam homofobia e construtos correlatos (Costa, Bandeira, \& Nardi, 2013b). A validade de critério para o preconceito foi confirmada pela comparação com a escala de Distância Social de Bogardus (Rodrigues, 2009). O instrumento é composto por 18 assertivas cujas respostas são distribuídas em uma escala Likert de 5 pontos $(1=$ discordo totalmente; 5 = concordo totalmente). A avaliação de fidedignidade dos itens da escala apresentou um alfa de Cronbach de 0,93 . No presente estudo, o escore total da escala foi calculado por somatório e o valor do alfa de Cronbach apresentado foi de 0,83 .

\section{Procedimentos}

Um questionário online contendo os instrumentos previamente descritos foi construído na plataforma Formulários Google e disponibilizado por 46 dias, entre 15 de março de 2016 e 29 de abril de 2016. Para garantir a leitura e compreensão do Termo de Consentimento Livre e Esclarecido (TCLE), foi criada uma subseção na qual se encontrava a única caixa de diálogo marcada como obrigatória. Para respeitar o direito de desistência de participantes, além da opção "Sim, eu li e compreendi o TCLE e aceito participar desta pesquisa", foi incluído "Não aceito participar desta pesquisa" - levando ao fim do questionário. Em caso de dúvida, participantes foram instruídos a contatar o pesquisador responsável. Foram informados os benefícios da participação, na colaboração com o progresso científico e na oportunidade de expressar opiniões, bem como os riscos de eventual desconforto com algum tema. No último caso, participantes poderiam, além de abandonar a pesquisa sem prejuízo, entrar em contato com o pesquisador responsável para comunicar a razão do desconforto.

\section{Considerações éticas}

Foram atendidas as diretrizes e normas para pesquisas envolvendo seres humanos do Conselho Nacional de Saúde (CNS), de acordo com as Resoluções $n^{\circ} 466 / 2012$ e n ${ }^{\circ} 510 / 2016$. O projeto foi aprovado pelo Comitê de Ética em Pesquisa da Universidade do Vale do Rio dos Sinos e pode ser conferido por meio do Certificado de Apresentação para Apreciação (CAAE) $\mathrm{n}^{\circ}$ 51286115.6.0000.5344.

\section{Análise dos dados}

As respostas do questionário online armazenadas em uma planilha de dados foram exportadas ao Statistical Package for the Social Sciences (SPSS 23.0). Para as análises, adotou-se $a$ priori a taxa de erro do Tipo I de 0,05 . Após exploração inicial dos dados, foram calculadas frequências e realizadas análises descritivas. Testes não paramétricos foram utilizados para comparações entre grupos sociodemográficos em relação ao preconceito, dada a distribuição anormal das variáveis. Utilizou-se o teste de Mann-Whitney para comparação entre grupos conforme gênero e orientação sexual e o teste de Kruskal-Wallis para comparação por local de moradia. A associação entre preconceito e variáveis tais como idade, perspectiva teórica, modalidade de trabalho e área de atuação foram analisadas por correlação de Spearman. Por fim, a análise de regressão linear múltipla, pelo método stepwise foi utilizada para calcular o valor preditivo de crenças sobre homossexualidade, bissexualidade e transexualidade na ocorrência de preconceito contra diversidade sexual e de gênero.

\section{Resultados}

A média do preconceito extremo contra diversidade sexual e de gênero entre os participantes da pesquisa foi de 1,46 $(D P=0,44)$, sendo o valor mínimo 1 e o máximo 5. Participantes heterossexuais apresentaram médias significativamente mais altas de preconceito que não heterossexuais (postos médios de 255,23 e 204,51, respectivamente; $U=13120,50 ; p<$ $0,01)$. Não foram encontradas diferenças significativas de preconceito entre gênero feminino e masculino ( $U=18995 ; p=0,57$ ) e local de moradia no interior, região metropolitana e capital $(H(2)=0,118 ; p=0,94)$. Ressalta-se que a variável gênero foi assumida como dicotômica somente porque entre os/as participantes havia apenas duas pessoas autodeclaradas não binárias, o que tornaria impossível formar uma terceira classe para comparação. Também não foi encontrada correlação significativa para idade $(p=1,188)$.

Analisando a relação entre o preconceito e o quanto cada uma das 10 perspectivas teóricas fundamentava $o$ trabalho do/a participante naquele momento, encon- 
trou-se correlação positiva significativa somente para "psicanálise e orientação psicanalítica", embora com um tamanho de efeito baixo $(\rho=0,11 ; p<0,05)$. Da mesma forma, os respondentes indicaram o quanto cada uma das oito modalidades de trabalho listadas correspondia à sua prática naquele momento. Apenas duas correlações significativas foram encontradas, embora ambas de baixa magnitude: "avaliação psicológica e/ou psicodiagnóstico" esteve positivamente correlacionada ao preconceito $(\rho=0,15 ; p<0,01)$ e "psicoterapia em grupo" apresentou correlação negativa ( $\rho=$ $-0,13 ; p<0,05)$. Já em relação à área de atuação, a Tabela 2 mostra correlações entre o preconceito e as 21 áreas listadas. Foram encontradas correlações positivas com "neurociência do comportamento" e correlações negativas com "análise institucional", "Psicologia da saúde", "Psicologia política" e "Psicologia social e comunitária".

Tabela 2

Correlações de preconceito e área de atuação.

\begin{tabular}{lcc}
\hline Área de atuação & $\rho$ & $n$ \\
\hline Análise institucional & $-0,10^{*}$ & 345 \\
Neurociência do comportamento & $0,19^{* *}$ & 345 \\
Psicologia clínica & 0 & 453 \\
Psicologia da saúde & $-0,12^{*}$ & 361 \\
Psicologia das emergências e desastres & $-0,09$ & 334 \\
Psicologia do desenvolvimento humano & -0 & 354 \\
Psicologia do esporte & 0,05 & 333 \\
Psicologia do trânsito & 0,09 & 340 \\
Psicologia escolar e educacional & $-0,01$ & 354 \\
Psicologia experimental & 0,11 & 337 \\
Psicologia hospitalar & $-0,01$ & 345 \\
Psicologia jurídica ou forense & 0,03 & 342 \\
Psicologia na área das deficiências & 0 & 335 \\
Psicologia organizacional e do trabalho & 0,07 & 353 \\
Psicologia política & $-0,14^{*}$ & 339 \\
Psicologia social e comunitária & $-0,19^{* *}$ & 361 \\
Sexualidade humana & $-0,10$ & 346 \\
Uso abusivo de substâncias & $-0,04$ & 352 \\
\hline Nota. ${ }^{*} p<0,05$. & & \\
$*_{*} p<01$. & &
\end{tabular}

Em relação às crenças sobre a natureza da homossexualidade, bissexualidade e transexualidade, os itens que compunham o tipo de crença psicossocial foram os mais pontuados entre os/as profissionais. A Tabela 3 mostra que isso foi constatado nas crenças sobre homossexualidade $(M=3,7)$, bissexualidade $(M=3,8)$ e transexualidade $(M=3,7)$.

Os itens das escalas de crenças que melhor explicaram a ocorrência de preconceito para cada escala são apresentados na Tabela 4. Foram encontrados coeficientes de determinação múltipla satisfatórios para os três modelos de regressão: crenças sobre homossexualidade $\left(R=0,61 ; R^{2}=0,37 ; R_{\text {ajustado }}^{2}=0,36\right.$; $p<0,001)$, crenças sobre bissexualidade $\left(R=0,64 ; R^{2}=\right.$ 0,$\left.41 ; R_{\text {ajustado }}^{2}=0,41 ; p<0,001\right)$ e crenças sobre transexualidade $\left(R=0,70 ; R^{2}=0,49 ; R_{\text {ajustado }}^{2}=0,48 ; p<0,001\right)$.

Nos três modelos, estão presentes itens identificados como crenças psicológicas (perversão, má resolução de conflitos com figuras parentais e abusos sexuais sofridos na infância). No entanto, no modelo de crenças sobre a transexualidade, o item "abusos sexuais sofridos

Tabela 3

Crenças sobre homossexualidade, bissexualidade e transexualidade.

\begin{tabular}{|c|c|c|c|c|}
\hline & Crenças & $n$ & $M$ & $D P$ \\
\hline \multirow{5}{*}{ 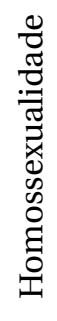 } & Biológicas & 497 & 1,5 & 0,8 \\
\hline & Religiosas & 497 & 1,0 & 0,3 \\
\hline & Ético-morais & 496 & 1,1 & 0,4 \\
\hline & Psicológicas & 497 & 1,9 & 1,2 \\
\hline & Psicossociais & 497 & 3,7 & 1,6 \\
\hline \multirow{5}{*}{ 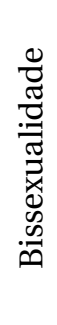 } & Biológicas & 497 & 1,5 & 0,7 \\
\hline & Religiosas & 496 & 1,0 & 0,3 \\
\hline & Ético-morais & 496 & 1,1 & 0,5 \\
\hline & Psicológicas & 497 & 1,8 & 1,1 \\
\hline & Psicossociais & 497 & 3,8 & 1,5 \\
\hline \multirow{5}{*}{ 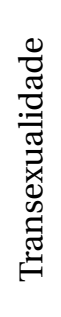 } & Biológicas & 497 & 1,6 & 0,9 \\
\hline & Religiosas & 496 & 1,0 & 0,3 \\
\hline & Ético-morais & 496 & 1,1 & 0,4 \\
\hline & Psicológicas & 497 & 1,8 & 1,1 \\
\hline & Psicossociais & 497 & 3,7 & 1,5 \\
\hline
\end{tabular}


Tabela 4

Crenças que melhor explicam a ocorrência de preconceito.

\begin{tabular}{|c|c|c|c|c|c|c|}
\hline & Modelo de crenças & $B$ & $\begin{array}{c}\text { Erro } \\
\text { Padrão }\end{array}$ & $\beta$ & $T$ & $p$ \\
\hline \multirow{6}{*}{ 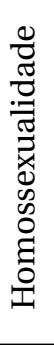 } & (Constante) & 9,28 & 1,56 & - & 5,93 & 0 \\
\hline & Perversão do comportamento sexual normal & 1,81 & 0,32 & 0,26 & 5,62 & 0 \\
\hline & Falta de fé religiosa & 7,50 & 1,30 & 0,25 & 5,76 & 0 \\
\hline & Má resolução de conflitos com as figuras parentais & 0,56 & 0,19 & 0,14 & 3,01 & 0 \\
\hline & Abusos sexuais sofridos na infância & 0,70 & 0,24 & 0,13 & 2,83 & 0 \\
\hline & Falta de caráter & 3,44 & 1,53 & 0,09 & 2,25 & 0,02 \\
\hline \multirow{6}{*}{ 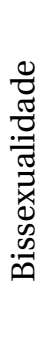 } & (Constante) & 11,41 & 1,17 & - & 9,77 & 0 \\
\hline & Perversão do comportamento sexual normal & 1,93 & 0,33 & 0,27 & 5,72 & 0 \\
\hline & Falta de fé religiosa & 6,02 & 0,87 & 0,27 & 6,91 & 0 \\
\hline & Má resolução de conflitos com as figuras parentais & 0,63 & 0,19 & 0,15 & 3,28 & 0 \\
\hline & Falta de caráter & 2,90 & 0,95 & 0,12 & 3,04 & 0 \\
\hline & Abusos sexuais sofridos na infância & 0,59 & 0,27 & 0,09 & 2,17 & 0,03 \\
\hline \multirow{5}{*}{ 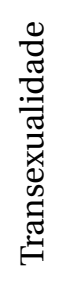 } & (Constante) & 11,92 & 1,09 & - & 10,90 & 0 \\
\hline & Perversão do comportamento normal de gênero & 2,93 & 0,30 & 0,43 & 9,98 & 0 \\
\hline & Falta de fé religiosa & 5,30 & 0,80 & 0,24 & 6,55 & 0 \\
\hline & Má resolução de conflitos com as figuras parentais & 0,62 & 0,17 & 0,14 & 3,62 & 0 \\
\hline & Falta de caráter & 2,84 & 0,94 & 0,11 & 3,02 & 0 \\
\hline
\end{tabular}

na infância" não apresentou impacto significativo no cálculo de regressão. Ressalta-se que o item "perversão" foi a variável com maior poder preditivo de preconceito em todos os modelos, seguida do item "falta de fé", que representa uma crença religiosa sobre a natureza da homossexualidade, bissexualidade e transexualidade.

\section{Discussão}

O preconceito contra diversidade sexual e de gênero entre os/as psicólogos/as da pesquisa apresentou uma média baixa em relação às pontuações da escala. No entanto, por ser uma medida de preconceito extremo, qualquer valor acima do mínimo pode ser considerado preocupante, especialmente tendo em vista a população estudada (Costa, Bandeira, \& Nardi, 2015b; Costa et al., 2015a).

Quanto às diferenças de preconceito em relação a variáveis sociodemográficas, neste estudo, confirmou-se apenas um dos consensos encontrados na literatura e apontados por Costa e Nardi (2015). Os resultados mostraram que psicólogos/as heterosse- xuais manifestaram mais preconceito do que os não heterossexuais. Na população geral, esse dado é confirmado por diversos estudos (Costa et al., 2015a,b; Dovidio, Hewstone, Glick, \& Esses, 2010). Isso pode ser explicado pela abordagem funcional das atitudes, na qual entende-se que as pessoas formam e mantêm atitudes em razão de benefício psicológico (Herek, \& McLemore, 2013). No caso de pessoas heterossexuais, atitudes positivas frente a pessoas não heterossexuais provavelmente aumentam na medida em que o preconceito não lhes traz mais benefícios psicológicos.

Já outras características comumente associadas com preconceito apresentaram resultados diferentes, incluindo gênero e local de moradia. De acordo com a literatura, homens são mais preconceituosos que mulheres (Cerqueira-Santos, Winter, Salles, Longo, \& Teodoro, 2007; Costa et al., 2015a,b; Gato, Fontaine, \& Leme, 2014) e pessoas que moram no interior têm mais preconceito que pessoas residentes em capitais ou regiões metropolitanas (Costa et al. 2015b). Isso não foi confirmado na presente pesquisa, pois não 
foram encontradas diferenças significativas referentes a essas variáveis. Considerou-se a possibilidade de características da profissão de psicólogo/a (por exemplo, empatia) terem amenizado a expressão de preconceito de homens e pessoas residentes no interior.

A crença em uma natureza psicossocial da homossexualidade, bissexualidade e transexualidade foi a mais frequente entre os/as psicólogos/as deste estudo. Ainda que se discorde que diversidade sexual e de gênero tenha uma "causa" determinante, objetivou-se investigar os modelos explicativos que as pessoas desenvolvem sobre a natureza de grupos sociais (Lacerda et al., 2002). No modelo utilizado neste estudo, as crenças psicossociais são representadas pelas seguintes atribuições causais: práticas culturais de cada sociedade, modo como se forma a identidade da pessoa e preferência do indivíduo por uma orientação sexual ou expressão de gênero. Pesquisas apontam que os menores níveis de preconceito estão associados com essas crenças psicossociais (Pereira et al., 2009a; 2011; 2014;). Portanto, considera-se um resultado positivo que a maioria dos/as psicólogos/ as atribuam causalidade a uma natureza psicossocial da homossexualidade, bissexualidade e transexualidade, em detrimento de crenças religiosas, ético-morais, biológicas e psicológicas. No entanto, ainda que a maioria dos/as psicólogos/as tenha relacionado a diversidade a uma natureza psicossocial, os modelos de regressão indicaram que atribuições causais que mais explicaram a ocorrência de preconceito foram as categorizadas como crenças psicológicas (perversão, má resolução de conflitos parentais e abusos sexuais sofridos na infância).

A atribuição causal que mais obteve impacto na ocorrência do preconceito foi considerar a homossexualidade e bissexualidade como perversão do comportamento sexual normal e a transexualidade como perversão do comportamento normal de gênero. Para autores como Ceccarelli (2010), essa abordagem seria perversa por si só, quando o discurso essencialista e os interesses da indústria farmacêutica nomeiam sexualidades como patologias, transformando o singular em anormal. Assim como a perversão, as atribuições causais de má resolução de conflitos com figuras parentais e abusos sexuais sofridos na infância também tiveram impacto nos maiores níveis de preconceito. Essas noções advêm de conhecimentos psicológicos que, principalmente, se originam na perspectiva psicanalítica e/ou psiquiátrica. Para Arán (2009), em seu modelo tradicional, a psicanálise se pauta na diferença sexual, na reiteração da heterossexualidade como norma e na dominação masculina e, portanto, precisa rever perspectivas em torno de temas como a positivação da feminilidade, as relações entre pessoas do mesmo gênero e o olhar lançado sobre pessoas trans. Além disso, grande parte das práticas clínicas em Psicologia, incluindo avaliação psicológica, está majoritariamente baseada em manuais médicos de doenças mentais, principalmente o Manual Diagnóstico de Doenças Mentais (DSM) e a Classificação Estatística Internacional de Doenças e Problemas Relacionados com a Saúde (CID).

Para a psiquiatria, a homossexualidade e a travestilidade eram consideradas como transtornos da personalidade sociopática, classificadas como desvios sexuais na primeira versão do DSM (American Psychiatric Association, 1952). A homossexualidade foi retirada da classificação de doenças mentais na segunda versão do DSM (American Psychiatric Association, 1973), após pressões de movimentos sociais. No entanto, os diagnósticos de gênero continuam sendo reclassificados a cada edição do manual, como "transexualismo" e "disforia de gênero" (DSM-III; American Psychiatric Association, 1980), "transtorno de identidade de gênero" (DSM-IV; American Psychiatric Association, 1994) e novamente "disforia de gênero" na quinta e última versão (American Psychiatric Association, 2013).

O mesmo acontece na CID. O termo "homossexualismo" foi retirado da décima versão, em 1990 (Organização Mundial da Saúde, 1997). Diagnósticos de gênero aparecem na oitava versão, com o termo "travestismo" considerado um desvio sexual (World Health Organization, 1965). O termo "transexualismo" surge em seguida, na nona versão do manual (Organização Mundial da Saúde, 1975) e é mantido no CID-10, no qual são incluídos "travestismo bivalente" e "transtornos de identidade de gênero". Discute-se, atualmente, sobre a modificação da próxima versão, que utilizará a nomenclatura "incongruência de gênero", provavelmente a ser publicada em 2018 (Beek et al., 2016).

As três atribuições causais que representam crenças psicológicas do instrumento estiveram/estão presentes nas abordagens tradicionais da Psicologia sobre sexualidade e gênero (Borges et al., 2013). Os dados corroboram a necessidade de inserir o tema da diversidade sexual e de gênero nos currículos oficiais da Psicologia, já que a produção teórica disponível se pauta majoritariamente em conhecimentos patologizantes.

Da mesma forma, as correlações encontradas entre preconceito e a perspectiva teórica da psicanálise e 
orientação psicanalítica, a modalidade de avaliação psicológica e/ou psicodiagnóstico e a área de atuação em neurociência do comportamento apoiam a necessidade de atenção aos conhecimentos disponíveis. Perspectivas teóricas, modalidades e áreas de atuação identificadas com uma Psicologia mais positivista produziram/produzem conhecimentos pautados em uma noção de normalidade, a qual produz também a noção de desvio ou patologia (Safatle, 2011). Por outro lado, a modalidade da psicoterapia em grupo, bem como as áreas de atuação em análise institucional, Psicologia da saúde, Psicologia política e Psicologia social e comunitária apresentaram associações com menores índices de preconceito. É possível que isso se explique pelos paradigmas adotados por profissionais dessa modalidade e dessas áreas. Posturas teórico-metodológicas que levem em conta os aspectos históricos, culturais e políticos da sexualidade e das expressões de gênero não somente tendem a estar associadas com baixo preconceito, mas também produzem formas menos excludentes de fazer Psicologia.

Por fim, embora não tenha sido frequente entre os/as psicólogos/as do estudo, crenças religiosas e ético-morais sobre a natureza da diversidade sexual e de gênero também explicaram a ocorrência do preconceito. Sabe-se que tais explicações não se fundamentam em teorias ou práticas psicológicas, no entanto, se o preconceito dos/as profissionais se sustenta nessas ideias, deve-se atentar para a permeabilidade da profissão às noções de sistemas explicativos não científicos. Trata-se, neste caso, de uma prática "pervertida", no sentido de que utiliza do lugar social da Psicologia para operar conhecimentos, valores e saberes religiosos ou de cunho moralista. Os acontecimentos recentes no país, referentes ao aviltamento da Resolução $\mathrm{n}^{\circ}$ 01/99 por parte de grupos com interesses políticos e religiosos, apontam para a constante necessidade de se examinar tais atravessamentos na ciência e profissão psicológica.

Advoga-se, a partir dos resultados deste estudo, pela construção de uma Psicologia menos estigmatizadora e preconceituosa, que contribua cada vez mais com uma mudança na direção da trajetória histórica de sua participação na opressão de pessoas não heterossexuais/cisgêneras. A mudança do foco de pesquisas seria um passo inicial, quando se deixa de investigar o "problema LGBT" e passa-se a examinar o preconceito contra essas pessoas, por exemplo. No entanto, este artigo também suscita a questão de quais efeitos pode ter produzido a pesquisa ao convocar psicólogos/as a indicarem suas crenças em relação à diversidade sexual e de gênero. Esse questionamento se faz necessário na medida em que parte do dispositivo que sustenta o preconceito é uma certa convocação aos/às profissionais de Psicologia a falar sobre o tema, como se, a partir de seu lugar social, certa autoridade lhes fosse dada a fazê-lo. Ao mesmo tempo, não convocar esses/as profissionais a falar sobre a heterossexualidade ou sobre a cisgeneridade implicitamente sustenta essas expressões de sexualidade que não necessitariam de explicação, pois fazem parte de um campo normativo da sexualidade.

\section{Conclusão}

Avaliou-se o preconceito contra diversidade sexual e de gênero entre os/as psicólogos/as e descobriu-se média baixa, mas preocupante por se tratar de uma medida de preconceito extremo, especialmente considerando os princípios de não discriminação e de combate a quaisquer formas de violência referentes à profissão de psicólogo/a. Psicólogos/as heterossexuais manifestaram mais preconceito que não heterossexuais e foram encontradas associações de maior preconceito com a perspectiva teórica da psicanálise, com a modalidade de avaliação psicológica e/ou psicodiagnóstico e com a área de atuação em neurociência do comportamento. Os/as profissionais têm mais crenças psicossociais sobre a homossexualidade, bissexualidade e transexualidade, mas aqueles que atribuem a diversidade a uma causa psicológica, ético-moral ou religiosa manifestaram mais preconceito extremo. Os dados alertam para problemas na formação em Psicologia e a necessidade da revisão de pressupostos teórico-metodológicos que reproduzem preconceitos historicamente construídos.

Entre as limitações deste estudo, deve-se considerar o viés proveniente da desejabilidade social inerente a medidas de autorrelato, bem como a características da amostra, tais como diretrizes que regulam o exercício profissional do/a psicólogo para a não expressão de preconceito. Também deve-se levar em conta a assimetria da distribuição da amostra por regiões do país, que embora semelhante à distribuição dos/as psicólogos no Brasil, em alguns casos, impede comparações. Sugere-se que futuros estudos investiguem, por meio de delineamentos mais complexos (por exemplo, experimentais ou quase-experimentais), como crenças e atitudes dos profissionais, bem como a formação e preparação que possuem, influenciam nas práticas psicológicas que desenvolvem nos mais variados contextos. 


\section{Referências}

American Psychiatric Association. (1952). Diagnostic and statistical manual of mental disorders. Washington, DC: o autor.

American Psychiatric Association. (1980). Diagnostic and statistical manual of mental disorders (3rd ed.). Arlington, VA: 0 autor.

American Psychiatric Association. (1994). Diagnostic and statistical manual of mental disorders (4th ed.). Arlington, VA: $o$ autor.

American Psychiatric Association. (2013). Diagnostic and statistical manual of mental disorders (5th ed.). Washington, DC: $\mathrm{o}$ autor.

American Psychiatric Association. (1973). Homosexuality and sexuality orientation disturbance: Proposed change in $D S M-I I$. Arlington, VA: o autor.

American Psychological Association. (2009). Report of the APA task force on gender identity and gender variance. Recuperado de https://www.apa.org/pi/lgbt/resources/policy/gender-identity-report.pdf

Arán, M. (2009). A psicanálise e o dispositivo diferença sexual. Revista Estudos Feministas, 17(3), 653-673. https:// doi.org/10.1590/s0104-026x2009000300002

Araújo, L. F., Oliveira, J. S. C., Sousa, V. C., \& Castanha, A. R. (2007). Adoção de crianças por casais homoafetivos: Um estudo comparativo entre universitários de Direito e de Psicologia. Psicologia \& Sociedade, 19(2), 95-102. https:// doi.org/10.1590/s0102-71822007000200013

Australian Psychological Society. (2007). Lesbian, gay, bisexual and transgender (LGBT) parented families: A literature review prepared for the Australian Psychological Society. Melbourne: o Autor.

Beek, T. F., Cohen-Kettenis, P. T., Bouman, W. P., Vries, A. L. C., Steensma, T. D., Witcomb, G. L. et al. (2016). Gender incongruence of adolescence and adulthood: Acceptability and clinical utility of the World Health Organization's proposed ICD-11 criteria. Plos One, 11(10). https://doi.org/10.1371/journal.pone.0160066

Borges, L. S., Canuto, A. A. A., Oliveira, D. P., \& Vaz, R. P. (2013). Abordagens de gênero e sexualidade na Psicologia: Revendo conceitos, repensando práticas. Psicologia: Ciência e Profissão, 33(3), 730-745. https://doi.org/10.1590/ s1414-98932013000300016

Brasil. (2011). Secretaria de Direitos Humanos. Relatório sobre violência homofóbica no Brasil:Ano de 2011. Brasília, DF: o autor.

Brasil. (2012). Secretaria de Direitos Humanos. Relatório sobre violência homofóbica no Brasil: Ano de 2012. Brasília, DF: o autor.

Camino, L., \& Pereira, C. R. (2000). O papel da Psicologia na construção dos direitos humanos: Análise das teorias e práticas psicológicas na discriminação ao homossexualismo. Perfil, 13(13), 49-69. https://doi.org/10.1590/ S0102-37722013000100010

Canadian Psychological Association. (n.d.). Policy and position statements. Ottawa: o autor. Recuperado de http:// www.cpa.ca/aboutcpa/policystatements

Ceccarelli, P. R. (2010). A nova ordem repressiva. Psicologia: Ciência e Profissão, 30(4), 738-751. https://doi. org/10.1590/S1414-98932010000400006

Cerqueira-Santos, E., Winter, F. D. S., Salles, L. A., Longo, J. L., \& Teodoro, M. (2007). Contato interpessoal e crenças sobre homossexualidade: Desenvolvimento de uma escala. Interação em Psicologia, 11(2), 221-229. https://doi. org/10.5380/psi.v1li2.6639

Conselho Federal de Psicologia. (2011). Psicologia e diversidade sexual: Desafios para uma sociedade de direitos. Brasília, DF: o autor.

Conselho Federal de Psicologia. (2017) Resolução CFP 01/99 é mantida em decisão judicial. Retrieved from http:// site.cfp.org.br/resolucao-cfp-0199-e-mantida-em-decisao-judicial

Conselho Federal de Psicologia. (n. d.). Despatologização das identidades trans e travestis. Recuperado de http:// despatologizacao.cfp.org.br 
Costa, A. B., Bandeira, D. R., \& Nardi, H. C. (2013b). Systematic review of instruments measuring homophobia and related constructs. Journal of Applied Social Psychology, 43(6), 1324-1332. https://doi.org/10.1111/jasp.12140

Costa, A. B., Bandeira, D. R., \& Nardi, H. C. (2015b). Avaliação do preconceito contra diversidade sexual e de gênero: Construção de um instrumento. Estudos de Psicologia, 32(2), 163-172. https://doi.org/10.1590/0103166X2015000200002

Costa, A. B., Machado, W. L., Bandeira, D. R., Nardi, H. C. (2016). Validation study of the revised version of the Scale of Prejudice Against Sexual and Gender Diversity in Brazil. Journal of Homosexuality, 63(11), 1446-1463. https://doi.org/10.1080/00918369.2016.1222829

Costa, A. B., \& Nardi, H. C. (2015). Homofobia e preconceito contra diversidade sexual: Debate conceitual. Temas em Psicologia, 23(3), 715-726. https:// doi.org/10.9788/tp2015.3-15

Costa, A. B., Peroni, R. O., Bandeira, D. R., \& Nardi, H. C. (2013a). Homophobia or sexism? A systematic review of prejudice against nonheterosexual orientation in Brazil. International Journal of Psychology, 48(5), 900-909. https:// doi.org/10.1080/00207594.2012.729839

Costa, A. B., Peroni, R. O., Camargo, E. S., Pasley, A., \& Nardi, H. C. (2015a). Prejudice toward gender and sexual diversity in a Brazilian public university: Prevalence, awareness, and the effects of education. Sexuality Research and Social Policy, 12(4), 261-272. https://doi.org/10.1007/s13178-015-0191-z

Costa, A. B., Santos, C. B., Rodrigues, M. C., \& Nardi, H. C. (2009). Attitudes among Brazilian university undergraduates regarding cinema with contents on sexual diversity. Journal of LGBT Youth, 6(4), 436-440. https://doi. org/10.1080/19361650903297070

Daniel, M., \& Baudry, A. (1977). Os homossexuais. Rio de Janeiro, RJ: Artenova.

Dovidio, J., Hewstone, M., Glick, P., \& Esses, V. (2010). The SAGE handbook of prejudice, stereotyping and discrimination. London, UK: SAGE.

Formiga, N. S. (2007). Valores humanos e sexismo ambivalente. Revista do Departamento de Psicologia - UFF, 19(2), 381-396. https://doi.org/10.1590/s0104-80232007000200009

Formiga, N. S., \& Silva Neta, A. B. (2009). Precisão preditiva das novas formas de sexismo a partir das orientações valorativas em brasileiros. Psico, 40(2), 174-183. Recuperado de http://revistaseletronicas.pucrs.br/fo/ojs/index. $\mathrm{php} /$ revistapsico/article/view/4038

Gato, J., Fontaine, A. M., \& Leme, V. B. R. (2014). Validação e adaptação transcultural da Escala Multidimensional de Atitudes Face a Lésbicas e a Gays. Psicologia: Reflexão e Crítica, 27(2), 257-271. https://doi.org/10.1590/16787153.201427206

Herek, G. M., \& McLemore, K. A. (2013). Sexual prejudice. Annual Review of Psychology, 64, 309-333. https://doi. org/10.1146/annurev-psych-113011-143826

Jesus, J. G. (2013). O conceito de heterocentrismo: Um conjunto de crenças enviesadas e sua permanência. Psico-USF, 18(3), 363-372. https://doi.org/10.1590/s1413-82712013000300003

Lacerda, M., Pereira, C. R., \& Camino, L. (2002). Um estudo sobre as formas de preconceito contra homossexuais na perspectiva das representações sociais. Psicologia: Reflexão e Crítica, 15(1), 165-178. https://doi.org/10.1590/ s0102-79722002000100018

Medin, D. L., \& Ortony, A. (1989). Psychological essentialism. In S. Vosniadou \& A. Ortony (Eds.), Similarity and analogical reasoning (pp. 179-195). Cambridge: Cambridge University Press.

Organização Mundial da Saúde. (1975). Classificação internacional de doenças: Adaptada para uso em processamento de dados. Porto Alegre, RS: Sagra.

Organização Mundial da Saúde. (1997). Classificação estatística internacional de doenças e problemas relacionados à saúde (10a rev.). São Paulo, SP: Universidade de São Paulo.

Pan American Health Organization. (n.d.). "Cures" for an illness that does not exist. Recuperado de http://www. paho.org/hq/index.php?option=com_docman\&task=doc_view\&gid=17703\&Itemid=270\&lang=en

Pereira, A. S. L. S., Alfaia, A. J. B., Souza, L. E. C., \& Lima, T. J. S. (2014). Preconceito contra homossexuais no contexto do futebol. Psicologia \& Sociedade, 26(3), 737-745. https:// doi.org/10.1590/s0102-71822014000300022 
Psicologia: Ciência e Profissão Out/Dez. 2018 v. 38 n4, 744-757.

Pereira, A. S. L. S., Monteiro, M. B., \& Camino, L. (2009b). Estudo da validação das escalas de crenças sobre a natureza da homossexualidade e de preconceito contra homossexuais. Laboratório de Psicologia, 7(1), 21-32. https:// doi.org/10.14417/lp.683

Pereira, A. S. L. S., Monteiro, M. B., \& Camino, L. (2009a). Social norms and prejudice against homosexuals. Spanish Journal of Psychology, 12(2), 576-84. https://doi.org/10.1017/s1138741600001943

Pereira, C. R., Torres, A. R. R., Pereira, A. S. L. S., \& Falcão, L. C. (2011). Preconceito contra homossexuais e representações sociais da homossexualidade em seminaristas católicos e evangélicos. Psicologia: Teoria e Pesquisa, 27(1), 73-82. https://doi.org/10.1590/S0102-37722011000100010

Pettigrew, T. F., \& Meertens, R. W. (2001). In defense of the subtle prejudice concept: A retort. European Journal of Social Psychology, 31(3), 299-309. https://doi.org/10.1002/ejsp.45

Pettigrew, T. F., \& Meertens, R. W. (1995). Subtle and blatant prejudice in Western Europe. European Journal of Social Psychology, 25(1), 57-75. https://doi.org/10.1002/ejsp.2420250106

Resolução No 001/99, de 22 de março de 1999. Estabelece normas de atuação para os psicólogos em relação à questão da orientação sexual. Brasília, DF: Conselho Federal de Psicologia.

Rodrigues, A. (2009). Psicologia social. Petrópolis, RJ: Vozes.

Rothbart, M., \& Taylor, M. (1992). Category labels and social reality: Do we view social categories as natural kinds? In G. Semin, \& K. Fiedler (Eds.), Language, interaction and social cognition (pp. 11-36). Newbury Park, CA: Sage.

Safatle, V. (2011). O que é uma normatividade vital? Saúde e doença a partir de Georges Canguilhem. Scientiae Studia, 9(1), 11-27. https://doi.org/10.1590/S1678-31662011000100002

Shipherd, J. C. (2015). Defining competence when working with sexual and gender minority populations: Training models for professional development. Clinical Psychology: Science and Practice, 22(2), 101-104. https://doi. org/10.1111/cpsp.12100

Sposito, S. H. (2015). Homossexualidades nas pesquisas em pós-graduação em psicologia: Da despatologização à luta por direitos (Doutorado). Universidade Estadual Paulista São Paulo, SP, Brasil.

World Health Organization. (1965). International classification of diseases (8th rev.). Retrieved from http://www. wolfbane.com/icd/icd8.htm

World Health Organization. (2012). "Therapies” to change sexual orientation lack medical justification and threaten health. Retrieved from http://www.paho.org/sur/index.php?option=com_content\&view=article\&id=414\&Ite$\operatorname{mid}=400$

Icaro Bonamigo Gaspodini

Graduação em Psicologia pela Faculdade Meridional (IMED), Passo Fundo - RS. Brasil. Mestre e doutorando em Psicologia pela Universidade do Vale do Rio dos Sinos (Unisinos), São Leopoldo - RS. Brasil. Bolsista Capes/Prosup. Professor do Curso de Psicologia da Faculdade Meridional IMED.

E-mail: icaroicaro@gmail.com

\section{Denise Falcke}

Graduação em Psicologia pela Pontifícia Universidade Católica do Rio Grande do Sul (PUC-RS), Porto Alegre - RS. Brasil. Especialização em Terapia de Casal e Família pelo Instituto de Terapias Integradas (ITI). Mestre e doutora pela PUC-RS. Professora adjunta do Programa de Pós-Graduação em Psicologia da Universidade do Vale do Rio dos Sinos (Unisinos), São Leopoldo - RS. Brasil.

E-mail:dfalcke@unisinos.br

Endereço para correspondência:

Av. Unisinos, 950, Setor E, Prédio 01 - Cristo Rei, São Leopoldo - RS, CEP 93020-190 
Recebido 19/05/2017

Reformulado 30/11/2017

Aprovado 20/02/2018

Received 05/19/2017

Reformulated $11 / 30 / 2017$

Approved 02/20/2018

Recibido 19/05/2017

Reformulado $30 / 11 / 2017$

Aceptado 20/02/2018

Como citar: Gaspodini, I. B., \& Falcke, D. (2018). Relações entre preconceito e crenças sobre diversidade sexual e de gênero em psicólogos/as brasileiros/as. Psicologia: Ciência e Profissão, 38(4), 744-757.

https://doi.org/10.1590/1982-3703001752017

How to cite: Gaspodini, I. B., \& Falcke, D. (2018). Relations between prejudice and beliefs about sexual and gender diversity among Brazilian psychologists. Psicologia: Ciência e Profissão, 38(4), 744-757. https://doi.org/10.1590/1982-3703001752017

Cómo citar: Gaspodini, I. B., \& Falcke, D. (2018). Relaciones entre preconcepto y creencias sobre diversidad sexual y de género en psicólogos/as brasileños/as. Psicologia: Ciência e Profissão, 38(4), 744-757. https://doi.org/10.1590/1982-3703001752017 